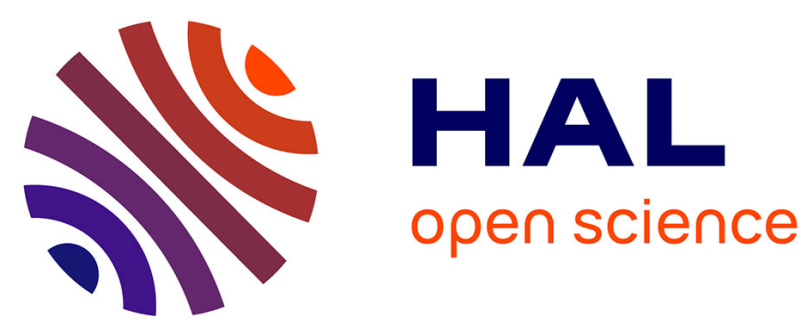

\title{
Exploratory analysis of a neutron-rich nuclei source based on photo-fission
}

\author{
M. Mirea, O. Bajeat, F. Clapier, S. Essabaa, L. Groza, F. Ibrahim, S. \\ Kandri-Rody, A-C. Mueller, N. Pauwels, J. Proust
}

\section{- To cite this version:}

M. Mirea, O. Bajeat, F. Clapier, S. Essabaa, L. Groza, et al.. Exploratory analysis of a neutron-rich nuclei source based on photo-fission. Nuclear Instruments and Methods in Physics Research Section B: Beam Interactions with Materials and Atoms, 2003, 201, pp.433-448. in2p3-00019858

\section{HAL Id: in2p3-00019858 https://hal.in2p3.fr/in2p3-00019858}

Submitted on 24 Mar 2003

HAL is a multi-disciplinary open access archive for the deposit and dissemination of scientific research documents, whether they are published or not. The documents may come from teaching and research institutions in France or abroad, or from public or private research centers.
L'archive ouverte pluridisciplinaire HAL, est destinée au dépôt et à la diffusion de documents scientifiques de niveau recherche, publiés ou non, émanant des établissements d'enseignement et de recherche français ou étrangers, des laboratoires publics ou privés. 


\title{
Exploratory analysis of a neutron-rich nuclei source based on photo-fission
}

\author{
M. Mirea ${ }^{1,2}$, O. Bajeat ${ }^{1}$, F. Clapier ${ }^{1}$, S. Essabaa $^{1}$, \\ L. Groza ${ }^{2}$, F. Ibrahim ${ }^{1}$, S. Kandri-Rody ${ }^{1,3}$, A.C. Mueller ${ }^{1}$, \\ N. Pauwels ${ }^{4}$ and J. Proust ${ }^{1}$
}

\author{
${ }^{1}$ Institut de Physique Nucléaire, 91406 Orsay Cedex, France \\ ${ }^{2}$ Institute of Physics and Nuclear Engineering, P.O. Box MG-6, Bucarest, Romania \\ ${ }^{3}$ Université Chouaib Doukkali, BP 20 El Jadida, Maroc \\ ${ }^{4}$ Laboratoire d'Utilisation du Rayonnement Electromagnétique, 91898, Orsay Cedex, France
}

\begin{abstract}
A source of neutron rich ions can be conceived through the photo-fission process. An exploratory study of such a source is realized. A survey of the radiative electron energy loss theory is reported in order to estimate numerically the bremsstrahlung production of thick targets. The resulted bremsstrahlung angular and energy theoretical distributions delivered from $\mathrm{W}$ and $\mathrm{UCx}$ thick converters are presented and compared with previous results. Some quantities as the number of fission events produced in the fissionable source and the energy loss in the converters are also reported as function of the geometry of the combination and the incident electron energy. An attempt of comparison with experimental data shows a quantitative agreement. This study is focussed on initial kinetic energies of the electron beam included in the range 30-60 MeV, suitable for the production of large radiative $\gamma$-ray yields able to induce the ${ }^{238} \mathrm{U}$ fission through the giant dipole resonance. A confrontation with the number of fission events produced in the frame of the fast neutron induced fission method indicates that the photo-fission can be a competitive concept.
\end{abstract}

PACS No: 25.85.Jg Photofission, 29.25.-t Particle sources and targets

\section{Introduction}

Nuclear fission of heavy nuclei is a process which allows the production of neutron rich isotopes. Exploiting this property, a source of intermediate energy nuclei near the stability limit can be conceived using the ISOL (Isotope Separation On-Line) technique. The feasibility study of an exotic nuclei source involving the fission process represents 
the main objective of the PARRNe (Production d'Atomes Radioactif Riches en Neutrons) R\&D project. Two different concepts have been studied to build such a source. The first one [1], is based on the neutron induced fission of ${ }^{238} \mathrm{U}$. A high neutron flux can be produced by using the deuteron break-up reaction. Experimental and theoretical results concerning the analysis of this concept are reported in Refs. $[2,3,4]$. Secondly, in a competitive manner, it is intended to study the feasibility of an exotic nuclei source by involving the bremsstrahlung induced fission following the conceptual mechanism presented in Ref. [5]. In a first step, an electron beam of $30-60 \mathrm{MeV}$ is focussed onto a $\mathrm{W}$ converter or onto the uranium carbide target ( $\mathrm{UCx}$ ) itself to deliver a bremsstrahlung radiation. In the second step, the gamma rays induce the fission of the ${ }^{238} \mathrm{U}$. Large fission events yields are expected due to the huge photo-fission cross section traduced by a broad peak of about $160 \mathrm{mb}$ height for $15 \mathrm{MeV}$ gamma rays [6, 7]. Another peak for the $\Delta$ photo-fission resonance can be seen [8] for gamma rays of about $300 \mathrm{MeV}$, but, for our practical applications, the fission events produced by excitations energies beyond $60 \mathrm{MeV}$ are not significant. To realize such an experimental combination, a knowledge of the bremsstrahlung spectrum is essential. During their passage in the matter, the electrons radiate electromagnetic radiation easily because they have a large value of charge to mass ration and hence are subject to violent accelerations in atomic Coulomb fields. This work begins with an evaluation of bremsstrahlung distributions in order to serve several purposes: estimating the electron energy loss in the converter, estimating the neutron rich nuclei yields in the target, to compare the fission events productivities between two different experimental configurations (with or without converter) and to analyze the advantages or disadvantages in comparison with the concept based on fast neutron induced fission. Since the experimental systematic of bremsstrahlung spectra is not accurate, and the coverage is too sparse, one must rely on the theoretical results. The next section is devoted to this subject.

\section{Theoretical guideline}

In this section, the main steps used to deduce the bremsstrahlung emission from targets are overviewed. The distributions of the electrons inside a target are obtained using single and multiple scattering theories. The differential bremsstrahlung emission is determined by selecting formulas and correction factors that give the best estimate for the cross section. The angular distribution of the gamma rays is finally deduced by 
applying the folding theorem between the angular electron spread and the differential bremsstrahlung cross section. The slowing down of the electrons in matter is estimated to obtain the mean energy along the range. The spread of the electron energy is evaluated using an approximate expression for the Landau distribution. The double differential bremsstrahlung cross section in gamma energy and angle is obtained for different values of the electron straggling and depths in the target. Integrating the irradiated flux along the electron range, the absorption of the continuous bremsstrahlung in the converter (or in the target) is taken into account. The shape of the converter is considered as cylindrical. Intensities of second-generation photon spectra are produced only up to $10 \%$ of the emitted radiation with small energies which are not sufficient to produce gamma rays soft enough to induce fission [9], so, this effect is disregarded. Several authors attack in slightly different ways the phenomena associated to the bremsstrahlung, less or more accurately. This situation motivated us to display the formulas used in the present work.

\subsection{Electron distributions}

The angular and the projected distributions of the electrons at a depth $d$ inside the target are obtained following the prescriptions of Ref. [10] concerning the validity of the Mott's cross section and the Molière's distributions. Therefore, if the depth where the electron distribution is determined is very small, that means the condition $d<<\frac{1}{\sigma N}$ is fulfilled (where $\sigma$ is the cross section and $N$ the number of atoms per volume unity), we use the Mott cross section:

$$
\frac{\mathrm{d} \sigma}{\mathrm{d} \Omega}=\frac{Z^{2} r_{0}^{2}}{4 \sin ^{4} \frac{\theta}{2}} \frac{1}{E^{2}\left(1-\frac{1}{E^{2}}\right)} R(E, Z, \theta) r(E, Z, \theta)
$$

where $R(E, Z, \theta)$ is the ratio between the cross section at high energy and the Rutherford's classical Coulomb scattering cross section, $r(E, Z, \theta)$ denotes a correction due to the shielding, $r_{0}=2.8 \times 10^{-13} \mathrm{~cm}$ is the classical electron radius and $E$ is the total energy of the electron in $m c^{2}$ units. The ratio $R(E, Z, \theta)$ is calculated with the model of Ref. [11] while values for $r(E, Z, \theta)$ are extracted from the literature [12]. Throughout this paper, $Z$ and $A$ denotes the atomic and the mass numbers of the converter. The probability that the electron is not deviated up to the depth $d$ is:

$$
P(\theta=0, d)=1-2 \pi N d \int_{0}^{\pi} \frac{\mathrm{d} \sigma}{\mathrm{d} \Omega} \sin \theta \mathrm{d} \theta
$$


In this domain, the differential projected distribution $W(\rho, d, z)$ can be deduced:

$$
W(\rho, d, z) \rho \mathrm{d} \rho=2 \pi N \frac{\mathrm{d} \sigma\left(\theta_{\rho}\right)}{\mathrm{d} \Omega} \frac{\cos \theta_{\rho}}{\rho^{2}+(d-z)^{2}} \mathrm{~d} z
$$

where

$$
\theta_{\rho}=\arctan \left(\frac{\rho}{d-z}\right)
$$

In the previous relations, $W(\rho, d, z)$ represents the probability that the electron deviates from its original direction (where the cylindrical coordinates $\rho=0$ ) to attain the ring surface $2 \pi \rho \mathrm{d} \rho$ around the initial direction, situated at the depth $d$ due to a scattering along a small portion of the range $\mathrm{d} z$. Therefore, the probability $W\left(\theta_{0}, d\right)$ to obtain the electron in an angular interval $\mathrm{d} \theta_{0}$ measured from the entrance side $(z=0)$ of the converter at the depth $d$ becomes by integration and by changing the variables:

$$
W\left(\theta_{0}, d\right)=2 \pi d^{2} \frac{\tan \theta_{0}}{\cos ^{2} \theta_{0}} \int_{0}^{d} N \frac{\mathrm{d} \sigma\left[\arctan \left(\frac{\rho}{d-x}\right)\right]}{\mathrm{d} \Omega} \frac{\cos \left[\arctan \left(\frac{\rho}{d-x}\right)\right]}{\rho^{2}+(d-z)^{2}} \mathrm{~d} z
$$

This last relation allows the determination of the lateral distribution of the electrons at different depths in the target.

If the depth $d$ becomes greater or equal to $\frac{1}{\sigma N}$, the multiple scattering of Molière [12] can be used. In the framework of this theory, the averaged angular distribution of multiple scattering is:

$$
F(\theta, d)=\frac{1}{2 \pi}\left[2 \exp \left(-\eta^{2}\right)+\frac{1}{B} F^{(1)}(\eta)+\frac{1}{B^{2}} F^{(2)}(\eta)\right]
$$

with $\eta=\theta /\left(\theta_{1} \sqrt{B}\right)$ being an reduced angle, $\theta_{1}=0.3965 Z(Z+1) /\left(m c^{2} p \beta\right) \sqrt{\rho d / A}, \rho$ is the density, $p$ is momentum in $m c$ unit, $m c^{2}=0.51 \mathrm{MeV}$, the parameter $B$ is the solution of the equation

$$
\ln B-B+\ln \gamma-0.154=0
$$

where $\gamma=8.831 \times 10^{3} q(Z+1) Z^{1 / 3} \rho d /\left(\beta^{2} A \Delta\right), \Delta=1.13+32 Z^{2} /(137 \beta)$. Values for the parameters $p$ and $\beta$ are obtained with the formulas given in the next subsection. The normalization condition

$$
2 \pi \int_{0}^{\pi / 2} F(\theta, d) \sin \theta \mathrm{d} \theta=1
$$

must be satisfied. This last condition in the small angle approximation reads:

$$
2 \pi \int_{0}^{\infty} F(\theta, d) \theta \mathrm{d} \theta=1
$$


For multiple scattering, the projected distribution $f$ as function of an angle $\theta_{0}$ measured from the origin ( $z=0$, entrance side of the target) is:

$$
f\left(\theta_{0}, d\right)=\frac{2}{\sqrt{\pi}} \exp \left[-\left(\frac{\theta_{0}}{\eta B^{1 / 2}}\right)^{2}\right]+\frac{1}{B} f^{(1)}\left(\frac{\theta_{0}}{\eta B^{1 / 2}}\right)+\frac{1}{B^{2}} f^{(2)}\left(\frac{\theta_{0}}{\eta B^{1 / 2}}\right)
$$

with the condition

$$
\int_{0}^{\infty} f\left(\theta_{0}, d\right) \mathrm{d} \theta_{0}=1
$$

Values for $F^{(i)}$ and $f^{(i)}(i=1,2)$ are tabulated in the literature [12].

An interpolation is realized in the plural scattering domain between the expressions deduced for single scattering and multiple scattering.

\subsection{Bremsstrahlung distributions}

The choice of the bremsstrahlung differential cross-section in photon energy and angle formulas as function of the electron energy was guided by the prescriptions given by [13] and [14]:

$$
\frac{\mathrm{d}^{2} \sigma}{\mathrm{d} k \mathrm{~d} \Omega}=\left\{\begin{array}{l}
\mathcal{A} f_{E} \frac{\mathrm{d}^{2} \sigma^{2 \mathrm{BN}}}{\mathrm{d} k \mathrm{~d} \Omega} \text { for } E_{e}<2 \mathrm{MeV} \\
\mathcal{A} \frac{\mathrm{d}^{2} \sigma^{2 \mathrm{BN}}}{\mathrm{d} k \mathrm{~d} \Omega} \text { for } 2 \mathrm{MeV}<E_{e}<50 \mathrm{MeV} \text { and } \gamma \geq 15 \\
\mathcal{A} \frac{\mathrm{d}^{2}{ }^{2 \mathrm{BS}}}{\mathrm{d} k \mathrm{~d} \Omega} \text { for } 2 \mathrm{MeV}<E_{e}<50 \mathrm{MeV} \text { and } \gamma<15 \\
\frac{\mathrm{d}^{2} \sigma^{2 \mathrm{SN}}}{\mathrm{d} k \mathrm{~d} \Omega} \text { for } 50 \mathrm{MeV}<E_{e}<100 \mathrm{MeV} \text { and } \gamma \geq 15 \\
\frac{\mathrm{d}^{2} \sigma^{2} \mathrm{~S}}{\mathrm{~d} k \mathrm{~d} \Omega} \text { for } 50 \mathrm{MeV}<E_{e}<100 \mathrm{MeV} \text { and } \gamma<15
\end{array}\right.
$$

where $E_{e}$ is the electron kinetic energy. Here, we used the Born approximation cross section $2 \mathrm{BN}$ and $2 \mathrm{BS}$ formulas and the relativistic cross section $2 \mathrm{CS}$ formulas with Coulomb correction. The 2BS formula is:

$\frac{\mathrm{d}^{2} \sigma^{2 \mathrm{BS}}}{\mathrm{d} k \mathrm{~d} \Omega}=\frac{4 Z^{2} r_{0}^{2}}{137 k} \frac{y E}{2 \pi \sin \theta}\left\{\frac{16 y^{2} E^{\prime}}{\left(y^{2}+1\right)^{4} E}-\frac{\left(E+E^{\prime}\right)^{2}}{\left(y^{2}+1\right)^{2} E^{2}}+\left[\frac{E^{2}+E^{\prime 2}}{\left(y^{2}+1\right)^{2} E^{2}}-\frac{4 y^{2} E^{\prime}}{\left(y^{2}+1\right)^{4} E}\right] \ln M(y)\right\}$

where,

$$
\begin{gathered}
y=E \theta \\
\frac{1}{M(y)}=\left(\frac{k}{2 E E^{\prime}}\right)^{2}+\left[\frac{Z^{1 / 3}}{111\left(y^{2}+1\right)}\right]^{2}
\end{gathered}
$$

The $2 \mathrm{BN}$ formula is

$$
\begin{gathered}
\frac{\mathrm{d}^{2} \sigma^{2 \mathrm{BN}}}{\mathrm{d} k \mathrm{~d} \Omega}=\frac{Z^{2} r_{0}^{2}}{8 \pi 137} \frac{1}{k} \frac{p^{\prime}}{p}\left\{\frac{8 \sin ^{2} \theta\left(2 E^{2}+1\right)}{p^{2} \Delta^{4}}-\frac{2\left(5 E^{2}+2 E E^{\prime}+3\right)}{p^{2} \Delta^{2}}-\frac{2\left(p^{2}-k^{2}\right)}{Q^{2} \Delta^{2}}+\frac{4 E^{\prime}}{p^{2} \Delta}+\right. \\
\frac{L}{p p^{\prime}}\left[\frac{4 E \sin ^{2} \theta\left(3 k-p^{2} E^{\prime}\right)}{p^{2} \Delta^{4}}+\frac{4 E^{2}\left(E^{2}+E^{\prime 2}\right)}{p^{2} \Delta^{2}}+\frac{2-2\left(7 E^{2}-3 E E^{\prime}+E^{\prime 2}\right)}{p^{2} \Delta^{2}}+\frac{2 k\left(E^{2}+E E^{\prime}-1\right)}{p^{2} \Delta}\right]- \\
\left.\left(\frac{4 \epsilon}{p^{\prime} \Delta}\right)+\left(\frac{\epsilon^{Q}}{p^{\prime} Q}\right)\left[\frac{4}{\Delta^{2}}-\frac{6 k}{\Delta}-\frac{2 k\left(p^{2}-k^{2}\right)}{Q^{2} \Delta}\right]\right\}
\end{gathered}
$$


where

$$
\begin{gathered}
L=\ln \left[\frac{E E^{\prime}-1+p p^{\prime}}{E E^{\prime}-1-p p^{\prime}}\right] \\
\Delta=E-p \cos \theta \\
\epsilon=\ln \left(\frac{E^{\prime}+p^{\prime}}{E^{\prime}-p^{\prime}}\right) \\
\epsilon^{Q}=\ln \left(\frac{Q+p^{\prime}}{Q-p^{\prime}}\right) \\
Q^{2}=p^{2}+k^{2}-2 p k \cos (\theta)
\end{gathered}
$$

The $2 \mathrm{CS}$ formula is

$$
\begin{gathered}
\frac{\mathrm{d}^{2} \sigma^{2 \mathrm{CS}}}{\mathrm{d} k \mathrm{~d} \Omega}=\frac{2 Z^{2} r_{0}^{2}}{137} \frac{1}{k} \frac{2 p u}{\left(1+u^{2}\right)} \frac{1}{2 \pi \sin \theta} \frac{1}{E^{2}} \times \\
\left\{\left(E^{2}+E^{\prime 2}\right)(3+2 \Gamma)-2 E E^{\prime}\left(1+4 u^{2} \epsilon^{2} \Gamma\right)\right\}
\end{gathered}
$$

where

$$
\begin{gathered}
\epsilon=\frac{1}{1+u^{2}} \\
u=p \theta \\
\Gamma=\ln \left(\frac{111 Z^{-1 / 3}}{\epsilon}\right)-2-f(Z) \\
f(Z)=\left\{\begin{array}{l}
1.2021(Z / 137)^{2} \text { for low } Z \\
0.925(Z / 137)^{2} \text { for high } Z
\end{array}\right.
\end{gathered}
$$

In the previous relations, $E$ is the total electron energy in $m c^{2}$ units, $E^{\prime}=E-k$ is the final energy of the electron after the emission of a quanta of energy $k, p$ and $p^{\prime}$ are the initial and final momentum of the electron in $m c$ units:

$$
\begin{gathered}
p=\sqrt{(E-1)(E+1)} \\
p^{\prime}=\sqrt{\left(E^{\prime}-1\right)\left(E^{\prime}+1\right)} \\
\beta=\frac{p}{E}
\end{gathered}
$$

Also, $\mathcal{A}$ represents a corrective factor for the Born approximation obtained by interpolating the data of Ref. [13], $f_{E}$ is the Elwert factor restricted to nonrelativistic electron energies:

$$
f_{E}=\frac{\beta\{1-\exp [-2 \pi Z /(137 \beta)]\}}{\beta^{\prime}\left\{1-\exp \left[-2 \pi Z /\left(137 \beta^{\prime}\right)\right]\right\}}
$$

and $\gamma=100 k\left(E E^{\prime} Z^{1 / 3}\right)^{-1}$ is a screening factor. 


\subsection{Folding the electron and gamma distributions}

The folding theorem is applied to obtain the angular distribution. As prescribed in Ref. [12], the folding theorem for spatial distributions reads:

$$
\begin{gathered}
F(\theta, \varphi)=F\left(\arctan \sqrt{\tan ^{2} \psi_{x}+\tan ^{2} \psi_{y}}, \arctan \frac{\tan \psi_{y}}{\tan \psi_{x}}\right)= \\
\int_{-\pi}^{\pi} \mathrm{d} \psi_{1 x} \int_{-\pi}^{\pi} \mathrm{d} \psi_{1 y} F_{1}\left(\arctan \sqrt{\tan ^{2} \psi_{1 x}+\tan ^{2} \psi_{1 y}}, \arctan \frac{\tan \psi_{1 y}}{\tan \psi_{1 x}}\right) \times \\
F_{2}\left[\arctan \sqrt{\tan ^{2}\left(\psi_{x}-\psi_{1 x}\right)+\tan ^{2}\left(\psi_{y}-\psi_{1 y}\right)}, \arctan \frac{\tan \left(\psi_{y}-\psi_{1 y}\right)}{\tan \left(\psi_{x}-\psi_{1 x}\right)}\right]
\end{gathered}
$$

where

$$
\begin{aligned}
& \tan \psi_{x}=\tan \theta \cos \varphi \\
& \tan \psi_{y}=\tan \theta \sin \varphi
\end{aligned}
$$

Due to the axial symmetry of the bremsstrahlung emission and angular electron distributions, the folding integral can be reduced to

$$
\begin{gathered}
\frac{\mathrm{d}^{2} \sigma_{f}(\theta)}{\mathrm{d} k \mathrm{~d} \Omega}=\int_{-\pi}^{\pi} \mathrm{d} \psi_{1 x} \int_{-\pi}^{\pi} \mathrm{d} \psi_{1 y} \frac{\mathrm{d}^{2} \sigma\left[\arctan \sqrt{\tan ^{2}\left(\psi_{x}-\psi_{1 x}\right)+\tan ^{2}\left(\psi_{y}-\psi_{1 y}\right.}\right)}{\mathrm{d} k \mathrm{~d} \Omega} \\
F\left(\arctan \sqrt{\tan ^{2} \psi_{1 x}+\tan ^{2} \psi_{1 y}}\right)+P(\theta=0, d) \frac{\mathrm{d}^{2} \sigma(\theta)}{\mathrm{d} k \mathrm{~d} \Omega}
\end{gathered} \times
$$

where the equality $\psi_{x}=\psi_{y}=\arctan (\tan \theta / \sqrt{2})$ is achieved in order to obtain a symmetric double folding integral, which is more suitable for a numerical treatment. Here, $P(\theta=0, d)$ denotes the probability that the electron is not deviated at the depth $d$ in the target (given by Rel. (2)). $P(\theta=0, d)$ is different from zero only in a thin

slice at the entrance side of the target. The quantity $\frac{\mathrm{d}^{2} \sigma_{f}(\theta)}{\mathrm{d} k \mathrm{~d} \Omega}$ is very sensitive on the variations of the electron distributions. Having in mind that the bremsstrahlung angular distribution falls several order of magnitude for an angular variation of $5^{\circ}$ around the incident direction, this distribution can be approximated with a delta function. In this last approximation, the angular distribution of the bremsstrahlung emission becomes that of the electron spread. To take into account the sudden variation of the bremsstrahlung cross section, the integral (31) is performed with 200 mesh points Gauss-Legendre quadratures. The rest of the integrals are performed simply with 32 mesh points Gauss-Legendre quadratures to avoid long computational times.

\subsection{Electron slowing down}

The slowing down of the electron is produced mainly by two effects: the inelastic collision and the bremsstrahlung production. The energy loss from ionization is proportional to $Z$ and increases only logarithmically with energy. The energy loss by radiation is proportional to $Z^{2}$ and increases linearly with the electron energy. The radiated energy 
appears as gamma-rays and forms the bremsstrahlung. The average value of the energy lost per unit path length is the stopping power of the absorbing substance.

The mean energy loss due to inelastic collisions is given by the following numerical formulas appropriate for low and high incident energies [10] The Bohr expression for the mean energy loss reads

$$
-\frac{\mathrm{d} \bar{E}_{c}}{\mathrm{~d} x}=0.306 \rho\left(\frac{Z}{A}\right) \beta^{-2} \ln \left(1.16 \frac{E_{k}}{I}\right)
$$

for $\beta<0.5$. The available Bethe - Bloch expression in the relativistic region is

$$
\begin{gathered}
-\frac{\mathrm{d} \bar{E}_{c}}{\mathrm{~d} x}=0.153 \rho \frac{Z}{A} \beta^{-2}\left[\ln \frac{E_{k}\left(E_{k}+m c^{2}\right)^{2} \beta^{2}}{2 I^{2} m c^{2}}+\left(1-\beta^{2}\right)-\right. \\
\left.2 \sqrt{1-\beta^{2}}-1+\beta^{2} \frac{1}{8}\left(1-\sqrt{1-\beta^{2}}\right)^{2}-\delta\right]
\end{gathered}
$$

for $\beta \geq 0.5$. The mean energy loss is given in $\mathrm{MeV} / \mathrm{cm}$, the density $\rho$ is considered in $\mathrm{g} / \mathrm{cm}^{3}$, here $E_{k}$ is the kinetic energy in $\mathrm{MeV}, m c^{2}=0.51 \mathrm{MeV}$, and $I$ are the ionization potentials which are listed in the literature [15]. The parameter $\delta$ is known as a density effect. The semi-empirical form of $\delta$ originally proposed in Ref. [16] is still used [15]:

$$
\delta=\left\{\begin{array}{l}
0 \text { for } X<X_{0} \\
4.606 X+C+a\left(X_{1}-X\right)^{m} \text { for } X_{0}<X<X_{1} \\
4.606 X+C \text { for } X_{1}<X
\end{array}\right.
$$

where

$$
X=\log _{10}\left(\frac{\beta}{\left(1-\beta^{2}\right)^{\frac{1}{2}}}\right)
$$

is a velocity dependent parameter,

$$
C=-2 \ln \left(\frac{I}{\hbar \omega_{p}}\right)-1
$$

is material dependent parameter, with $\omega_{p}^{2}=4 \pi N e^{2} / m$ being the bulk plasma frequency. Numerically $\hbar \omega_{p}=30.47\left(\frac{Z \rho}{A}\right)^{1 / 2}$ in eV. The density $\rho$ is expressed in $\mathrm{g} / \mathrm{cm}^{3}, m=3$,

$$
\begin{gathered}
a=-\left(C+4.606 X_{0}\right) /\left(X_{1}-X_{0}\right)^{m} \\
X_{0}=\left\{\begin{array}{l}
0.2 \text { for } I<100 \mathrm{eV} \text { and }|C|<3.681 \\
0.326|C|-1 \text { for } I<100 \mathrm{eV} \text { and }|C| \geq 3.681 \\
0.2 \text { for } I \geq 100 \mathrm{eV} \text { and }|C|<5.215 \\
0.326|C|-1.5 \text { for } I \geq 100 \mathrm{eV} \text { and }|C| \geq 5.215
\end{array}\right. \\
X_{1}=\left\{\begin{array}{l}
2 \text { for } I<100 \mathrm{eV} \text { and }|C|<3.681 \\
2 \text { for } I<100 \mathrm{eV} \text { and }|C| \geq 3.681 \\
3 \text { for } I \geq 100 \mathrm{eV} \text { and }|C|<5.215 \\
3 \text { for } I \geq 100 \mathrm{eV} \text { and }|C| \geq 5.215
\end{array}\right.
\end{gathered}
$$


The bremsstrahlung differential cross-section in photon energy formulas are obtained by using the prescriptions given in Ref. [13]. The same forms were used in the electron slowing down evaluations of Ref. [17]:

$$
\frac{\mathrm{d} \sigma}{\mathrm{d} k}=\left\{\begin{array}{l}
f_{E} \frac{\mathrm{d} \sigma^{3 \mathrm{BNa}} \mathrm{d} k}{\mathrm{~d} k} \text { for } 0.01 \mathrm{MeV}<E_{e}<0.1 \mathrm{MeV} \text { and } k \geq 0.01 T_{0} \\
\mathcal{A} f_{E} \frac{\mathrm{d} \sigma^{3 \mathrm{BN}}}{\mathrm{d} k} \text { for } 0.1 \mathrm{MeV} \leq E_{e}<2 \mathrm{MeV} \text { and } k \geq 0.01 T_{0} \\
\mathcal{A} \frac{\mathrm{d} \sigma^{3 \mathrm{BN}} \mathrm{d} k}{k} \text { for } 2 \mathrm{MeV} \leq E_{e}<15 \mathrm{MeV} \text { and } \gamma \geq 15 \\
\mathcal{A} \frac{\mathrm{d} \sigma^{3 \mathrm{BS}}}{\mathrm{d} k} \text { for } 2 \mathrm{MeV} \leq E_{e}<15 \mathrm{MeV} \text { and } 2<\gamma<15 \\
\mathcal{A} \frac{\mathrm{d} \sigma^{3 \mathrm{BSc}}}{\mathrm{d} k} \text { for } 2 \mathrm{MeV} \leq E_{e}<15 \mathrm{MeV} \text { and } \gamma \leq 2 \\
\frac{\mathrm{d} \sigma^{3 \mathrm{BN}}}{\mathrm{d} k} \text { for } 15 \mathrm{MeV} \leq E_{e}<50 \mathrm{MeV} \text { and } \gamma \geq 15 \\
\mathcal{A} \frac{\mathrm{d} \sigma^{3 \mathrm{BSd}}}{\mathrm{d} k} \text { for } 15 \mathrm{MeV} \leq E_{e}<50 \mathrm{MeV} \text { and } 2<\gamma<15 \\
\mathcal{A} \frac{\mathrm{d} \sigma^{3 \mathrm{BSc}}}{\mathrm{d} k} \text { for } 15 \mathrm{MeV} \leq E_{e}<50 \mathrm{MeV} \text { and } \gamma \leq 2 \\
\frac{\mathrm{d} \sigma^{3 \mathrm{BN}}}{\mathrm{d} k} \text { for } 50 \mathrm{MeV} \leq E_{e}<500 \mathrm{MeV} \text { and } \gamma \geq 15 \\
\frac{\mathrm{d} \sigma^{3 \mathrm{CSa}}}{\mathrm{d} k} \text { for } 50 \mathrm{MeV} \leq E_{e}<500 \mathrm{MeV} \text { and } 2<\gamma<15 \\
\frac{\mathrm{d} \sigma^{3 \mathrm{CSb}}}{\mathrm{d} k} \text { for } 50 \mathrm{MeV} \leq E_{e}<500 \mathrm{MeV} \text { and } \gamma \leq 2
\end{array}\right.
$$

where

$$
\begin{aligned}
& \frac{\mathrm{d} \sigma^{3 \mathrm{BNa}}}{\mathrm{d} k}=\frac{Z^{2} r_{0}^{2}}{137} \frac{16}{3} \frac{1}{p^{2}} \ln \left(\frac{p+p^{\prime}}{p-p^{\prime}}\right) \frac{1}{k} \\
& p=\frac{\beta_{0}}{\left(1-\beta_{0}^{2}\right)^{1 / 2}} \\
& \frac{\mathrm{d} \sigma^{3 \mathrm{BN}}}{\mathrm{d} k}=\frac{Z^{2} r_{0}^{2}}{137} \frac{p^{\prime}}{p}\left[\frac{4}{3}-2 E E^{\prime}\left(\frac{p^{2}+p^{2}}{p^{2} p^{\prime 2}}\right)+\frac{\mathcal{E} E^{\prime}}{p^{3}}+\frac{\mathcal{E}^{\prime} E}{p^{\prime 3}}+\frac{\mathcal{E} \mathcal{E}^{\prime}}{p p^{\prime}}+\mathcal{L} \mathcal{U}\right] \frac{1}{k} \\
& \mathcal{E}=\ln \frac{E+p}{E-p} \\
& \mathcal{E}^{\prime}=\ln \frac{E^{\prime}+p^{\prime}}{E^{\prime}-p^{\prime}} \\
& \mathcal{L}=2 \ln \left(\frac{E E^{\prime}+p p^{\prime}-1}{k}\right) \\
& \mathcal{U}=\frac{8 E E^{\prime}}{3 p p^{\prime}}+k^{2} \frac{E^{2} E^{\prime 2}+p^{2} p^{\prime 2}}{p^{3} p^{\prime 3}}+\frac{k}{2 p p^{\prime}}\left[\left(\frac{E E^{\prime}+p^{2}}{p^{3}}\right) \mathcal{E}-\left(\frac{E E^{\prime}+p^{\prime 2}}{p^{\prime 3}}\right) \mathcal{E}^{\prime}+\frac{2 k E E^{\prime}}{p^{\prime 2} p^{2}}\right] \\
& \frac{\mathrm{d} \sigma^{3 \mathrm{BSd}}}{\mathrm{d} k}=\frac{4 Z^{2} r_{0}^{2}}{137}\left[1+\left(\frac{E^{\prime}}{E}\right)^{2}-\frac{2}{3} \frac{E^{\prime}}{E}\right]\left[\ln \frac{2 E E^{\prime}}{k}-\frac{1}{2}-c(\gamma)\right] \frac{1}{k} \\
& c(\gamma)=0.102 \exp (-0.151 \gamma)+0.47 \exp (-19.8 \gamma) \\
& \frac{\mathrm{d} \sigma^{3 \mathrm{BSc}}}{\mathrm{d} k}=\frac{4 Z^{2} r_{0}^{2}}{137}\left\{\left[1+\left(\frac{E^{\prime}}{E}\right)^{2}\right]\left[\frac{\phi_{1}(\gamma)}{4}-\frac{\ln Z}{3}\right]-\frac{2 E^{\prime}}{3 E}\left[\frac{\phi_{2}(\gamma)}{4}-\frac{\ln Z}{3}\right]\right\} \frac{1}{k} \\
& \phi_{1}(\gamma)=\phi_{2}(\gamma)+0.5 \exp (-2.31 \gamma)+0.12 \exp (-19.8 \gamma) \\
& \phi_{2}(\gamma)=20.14 \exp (-0.151 \gamma)
\end{aligned}
$$




$$
\begin{gathered}
\frac{\mathrm{d} \sigma^{3 \mathrm{CSa}}}{\mathrm{d} k}=\frac{4 Z^{2} r_{0}^{2}}{137}\left\{\left[1+\left(\frac{E^{\prime}}{E}\right)^{2}-\frac{2 E^{\prime}}{3 E}\right]\left[\ln \left(\frac{183}{Z^{1 / 3}}\right)-f(Z)\right]+\frac{E^{\prime}}{9 E}\right\} \frac{1}{k} \\
f(Z)=\left[\frac{1}{1+(Z / 137)^{2}}+0.20206\right]\left(\frac{Z}{137}\right)^{2} \\
\frac{\mathrm{d} \sigma^{3 \mathrm{CSc}}}{\mathrm{d} k}=\frac{4 Z^{2} r_{0}^{2}}{137}\left\{\left[1+\left(\frac{E^{\prime}}{E}\right)^{2}\right]\left[\frac{\phi_{1}(\gamma)}{4}-\frac{\ln Z}{3}-f(Z)\right]-\frac{2 E^{\prime}}{3 E}\left[\frac{\phi_{2}(\gamma)}{4}-\frac{\ln Z}{3}-f(Z)\right]\right\}
\end{gathered}
$$

The mean energy loss due to the radiative process is

$$
\frac{\mathrm{d} \bar{E}_{b}}{\mathrm{~d} x}=\frac{6.0249 \times 10^{23} m c^{2}}{A} \int_{0}^{E_{k}} k \frac{\mathrm{d} \sigma}{\mathrm{d} k} \mathrm{~d} k
$$

so that the total energy loss is given by the relation

$$
\frac{\mathrm{d} \bar{E}}{\mathrm{~d} x}=\frac{\mathrm{d} \bar{E}_{c}}{\mathrm{~d} x}+\frac{\mathrm{d} \bar{E}_{b}}{\mathrm{~d} x}
$$

Our numerical results agree within few percents with the previous published values [17]

\subsection{Energy straggling of electrons}

Previously, only the mean value of the stopping power was obtained. The fluctuations in energy loss produces fluctuations in range. An estimation of the electron beam straggling must be realized because in traversing a given thickness of the scattering medium, the electron undergo discrete energy loss. As mentioned previously, the spectral distribution of the electron energy loss of fast electrons is ruled by two main Coulomb interactions: (i) collisions with atomic electrons, the so called ionization processes and (ii) collisions with atomic nuclei, which produces the bremsstrahlung radiation. The variation in energy loss due to both type of collisions is commonly referred as energy straggling. In the following, $f\left(E_{i}, E_{f}, x\right)$ refers to the energy loss straggling distribution in $\mathrm{MeV}^{-1}$ units. An electron initially with kinetic energy $E_{i}$, after passing through a target of thickness $x$, will have a probability $f\left(E_{i}, E_{f}, x\right) \mathrm{d} E_{f}$ of being in the kinetic energy interval between $E_{f}$ and $E_{f}+\mathrm{d} E_{f}$. Due to strong dependence of the bremsstrahlung cross section on the electron energy, it is important to take into account the energy straggling. The energy loss by ionization can be conveniently parametrized with the Landau straggling distribution. This distribution must be corrected to take into account the radiative processes. Within the approximation of the universal function appearing in the Landau theory reported in Ref. [18], this distribution can be represented as follows:

$$
f_{\text {ion }}\left(E_{i}, E_{f}, x\right)=\frac{1}{\xi} \varphi(\lambda)
$$


where

$$
\begin{gathered}
\xi=\frac{2 \pi m c^{2} N r_{0}^{2}}{\beta^{2}} \frac{Z}{A} x \\
\lambda=\frac{1}{\xi}\left\{E_{i}-E_{f}-\xi\left[\ln \left(\frac{2 \xi m c^{2} \beta^{2}}{(1-\beta)^{2} I^{2}}\right)-\beta^{2}+0.37-\delta\right]\right\} \\
\varphi(\lambda)=\left\{\begin{array}{cl}
\frac{a_{0}+a 1\left(\lambda-\lambda_{p}\right)^{2}}{1+b_{1}\left(\lambda-\lambda_{p}\right)^{2}+b_{2}\left(\lambda-\lambda_{p}\right)^{4}}, & \lambda_{1} \leq \lambda<\lambda_{p} \\
\frac{c_{0} d_{1}\left(\lambda-\lambda_{p}\right)+d_{2}\left(\lambda-\lambda_{p}\right)^{2}}{1+d_{1}}, & \lambda \leq \lambda \leq \lambda_{2}
\end{array}\right.
\end{gathered}
$$

Here $N=6.0249 \times 10^{23}$ is Avogadro's number, the classical electron radius is in $\mathrm{cm}$, $m c^{2}=0.51 \mathrm{MeV}$ the electron rest electron energy, $x$ is the depth in $\mathrm{g} / \mathrm{cm}^{2}$ (so that $\xi$ is in $\mathrm{MeV}$ units), $I$ is the ionization potential and $\delta$ the density effect. The parameters in the function $\varphi(\lambda)$ have the following values: $\lambda_{1}=-2.9, \lambda_{2} \rightarrow \infty, \lambda_{p}=-0.2225, a_{0}=0.17904$, $a_{1}=-0.0253, b_{1}=0.0482, b_{2}=0.1132, c_{0}=0.17904, d_{1}=0.0134$ and $d_{2}=0.1355$. This approximation reproduces the Landau formula within an absolute error of $2 \times 10^{-3}$.

The radiative energy loss straggling can be parametrized with the Bethe and Heitler formula. The form given in Ref. [19] was retained:

$$
f_{\mathrm{rad}}\left(E_{i}, E_{f}, x\right)=\frac{1}{\left(E_{i}+m c^{2}\right) \Gamma(l)}\left(\ln \frac{E_{i}+m c^{2}}{E_{f}+m c^{2}}\right)^{l-1}
$$

where $l=\frac{x}{X_{0} \ln 2}$ is the thickness in units of radiation length $X_{0}$. The radiation length can be obtained with the parameterization found in Ref. [20] which gives values in $\mathrm{g} / \mathrm{cm}^{2}$ :

$$
X_{0}=\frac{716.405 A}{Z^{2}\left(L_{r}-f\right)+z L_{r}^{\prime}}
$$

where $L_{r}=\ln \left(184.15 Z^{-1 / 3}\right), L_{r}^{\prime}=\ln \left(1194 Z^{-2 / 3}\right), f=1.202 z-1.0369 z^{2}+1.008 z^{2} /(1+$ $z$ ) and $z=(Z / 137)^{2}$.

The total probability that an electron of the initial energy $E_{i}$ reaches the energy $E_{f}$ while traversing a material of thickness $x$ is obtained by a folding procedure:

$$
f\left(E_{i}, E_{f}, x\right)=\int_{E_{f}}^{E_{i}} f_{\text {ion }}\left(E_{i}, y, x\right) f_{\text {rad }}\left(y, E_{f}, x\right) \mathrm{d} y
$$

This integral contains a singularity in the limit $y=E_{f}$ if $l<1$. To handle this problem in numerical computations, by following the prescriptions of Ref. [19], the folding integral is approximated with the expression:

$$
f\left(E_{i}, E_{f}, x\right)=\int_{y_{0}}^{E_{i}} f_{\text {ion }}\left(E_{i}, y, x\right) f_{\text {rad }}\left(y, E_{f}, x\right) \mathrm{d} y+f_{\text {ion }}\left(E_{i}, E_{f}, x\right) \frac{\left[\ln \left(\frac{y_{0}+m c^{2}}{E_{f}+m c^{2}}\right)\right]^{l}}{\Gamma(l+1)}
$$


where $y_{0}=\epsilon E_{f}$ and $\epsilon$ is a number slightly larger than one. In the present calculation $\epsilon=1.002$. Due to the presence of $m c^{2}$ in the formulas, the radiative distribution is not properly normalized:

$$
\int_{\mu}^{E_{i}} f\left(E_{i}, E_{f}, x\right) \mathrm{d} E_{f}=C
$$

so that the mean value of the energy is obtained by performing the renormalization with the constant $C$

$$
E_{s}=\frac{1}{C} \int_{0}^{E_{i}} E_{f} f\left(E_{i}, E_{f}, x\right) \mathrm{d} E_{f}
$$

This average energy is derived within the Landau distribution function which assumes that the ionization losses are small compared to the initial energy. Therefore, this kind of simulation does not take into account the attenuation of the electron beam due to energy loss comparable to the electron energy before collisions. In these circumstances, the average energy of the beam will be larger than that obtained within accurate formalism of the electron slowing down. To reproduce the average energy obtained with the theory used in computing the electron slowing down and to take into account the attenuation of the beam, the function distribution is multiplied with the ratio $r=\bar{E} / E_{s}$, so that our distribution function becomes $r f\left(E_{i}, E_{f}, x\right) . \bar{E}$ is the mean energy of the electron in the matter obtained with formula (56).

\subsection{Other effects}

As mentioned in Ref. [10], for thick foils theoretical calculations are very difficult and only for complete diffusion it is possible to give an analytical expression for the angular distribution

$$
F(\theta)=\frac{3}{4 \pi}(0.717+\cos \theta) \cos ^{2} \theta
$$

In order to improve the results at the end of the electron range, the angular distribution is varied linearly from that obtained with the multiple scattering formula to that available for complete diffusion. This linear variation begins when the electron average energy equals $1 / 4$ of the initial beam energy in order to reach the complete diffusion at the end of the electron range.

The endpoint region (high frequency) of the bremsstrahlung spectrum is corrected using the Fano formula [13]:

$$
\frac{\mathrm{d}^{2} \sigma}{\mathrm{d} \Omega \mathrm{d} k}=\frac{2 Z^{3} r_{0}^{2} \beta \sin ^{2} \theta}{137^{2} k^{3} E^{3}} \frac{1+\frac{1}{2} E(E-1)(E-2)(1-\beta \cos \theta)}{(1-\beta \cos \theta)^{4}}
$$


A linear interpolation is used in the way similar to that used in Ref. [14] in the region $0.85 T<k<T, T$ being the kinetic energy of the electron. The different cross section formulas are smoothly joined during the passage of their appropriate limits of validity by mean of a linear interpolation. This procedure avoids abrupt variations of the cross section in the vicinity of the limits of validity.

The effect of mixtures can be included in computing the lateral and angular deflections by replacing $N Z^{2}$ by $\sum_{i} N_{i} Z_{i}^{2}$ summed on different atomic species [12] $N_{i}$ being the number of atom of the specie $i$ in the volume unity. To compute the slowing down and the bremsstrahlung cross section, the effective values of the target parameters can be deduced using the Bragg rule [21, 22] which stipulates that the stopping of the individual elements are approximately additive. The same rule is valid for the gamma absorption coefficients [23]

The angular and energetic distributions are obtained by integrating the double bremsstrahlung differential cross section folded with the electron distribution along the range of the electron in the target, taking into account the gamma attenuation. For electromagnetic radiation the absorption is exponential. Energy is removed from the beam and lost in the target. The intensity of the gamma ray decreases with the thickness traversed, so that the final result depends on the geometric conditions of the experimental combination.

\section{Results}

The primary purpose of our calculations was to obtain the bremsstrahlung spectra of interest for the design of a neutron rich nuclei source based on the gamma induced fission of ${ }^{238} \mathrm{U}$. For that purpose, high gamma yields at energies around $15 \mathrm{MeV}$ are necessary, focussed as best as possible in the forward direction, in order to produce a large number of fission events in the fissionable target placed in the vicinity of the converter. A numerical tool was conceived to estimate these spectra. So, our analysis is mainly dedicated to small angles, where we need a good precision of our simulations, or, at least, an information about the degree of accuracy of our evaluation. In the following, the bremsstrahlung emissions of cylindrical $\mathrm{W}$ and $\mathrm{UCx}$ targets are simulated for initial energies of the electrons included in the interval 30-60 MeV. When the emerging spectra from targets are considered, in general the converter thicknesses are considered twice the range of the electron to allow comparisons with previous published results. 
Taking into consideration the Landau energy spread distribution, the differential cross section for the emission of a quanta $E_{\gamma}$ at the depth $z$ of the target will be:

$$
\frac{\mathrm{d}^{2} \sigma_{t}\left(\theta, E_{i}, z\right)}{\mathrm{d} E_{\gamma} \mathrm{d} \Omega}=\int_{E_{\gamma}}^{E_{i}} f_{\text {ion }}\left(E_{i}, E, z\right) \frac{\mathrm{d}^{2} \sigma_{f}(\theta, E, x)}{\mathrm{d} E_{\gamma} \mathrm{d} \Omega} \mathrm{d} E
$$

where the integration must be done for all non zero values of the energy distribution from the emitted gamma energy up to the incident electron energy. Here $\frac{\mathrm{d}^{2} \sigma_{f}}{\mathrm{~d} E_{\gamma} \mathrm{d} \omega}$ denotes the overlapped distribution between the electron angular distribution and that of the bremsstrahlung obtained with Rel. (31). We used the $f_{\text {ion }}$ distribution instead of $f$ given by Rel. (63) because when the radiative energy spread of the electron is taken into account, the behavior of $\mathrm{d}^{2} N_{\gamma} / \mathrm{d} E_{\gamma} \mathrm{d} \Omega$ (the differential number of photons emitted from a target of a certain geometry) remains approximately unchanged but falls in overall for about $50 \%$. In the same time, the results obtained with $f_{\text {ion }}$ are in better agreement with the data found in the literature, as those from Ref. [14] (considered as reference). However, this discrepancy has a possible explanation in the fact that, analyzing the text of Ref. [14], the energy loss in the path segments was sampled only from the Landau distribution, and afterwards added to the individual bremsstrahlung production events, without taking into consideration the radiative spread directly in the electron distribution. Therefore, it is possible that both kind of simulations overestimate the bremsstrahlung yields. Our calculations offer also a support for the confrontation of the different methods for the bremsstrahlung evaluation: the Monte Carlo one and the analytic one. Within the above defined cross section, the angular and energy distributions of the bremsstrahlung emitted from targets and converters can be obtained by integrating along the range $L$ of the electron path:

$$
\frac{\mathrm{d}^{2} N_{\gamma}}{\mathrm{d} E_{\gamma} \mathrm{d} \Omega}=\frac{1}{A u} \int_{0}^{L} \mathrm{~d} z \exp \left[-\mu\left(E_{\gamma}\right) d(\theta)\right] \frac{\mathrm{d}^{2} \sigma_{t}\left(\theta, E_{i}, z\right)}{\mathrm{d} E_{\gamma} \mathrm{d} \Omega}
$$

where $d$ denotes the distance in the material from the depth $z$ in the material to the exit point of the target (or converter), which depends on the emission angle $\theta$ of the quanta, $\mu$ is the attenuation coefficient of the mixture at an energy $E_{\gamma}, A$ is the weighted mass of the mixture, and $u$ the atomic mass unit. $N_{\gamma}$ is the number of gamma rays per electron. For the sake of simplicity of the formulae, we shall omit the integration over the electron lateral spread distribution. Details concerning the geometrical formulae applied in the numerical evaluations are available in Ref. [24]. The meaning of the geometric symbols can be identified by inspecting the Fig. 1 where a phenomenologic description of the phenomena is also presented. 


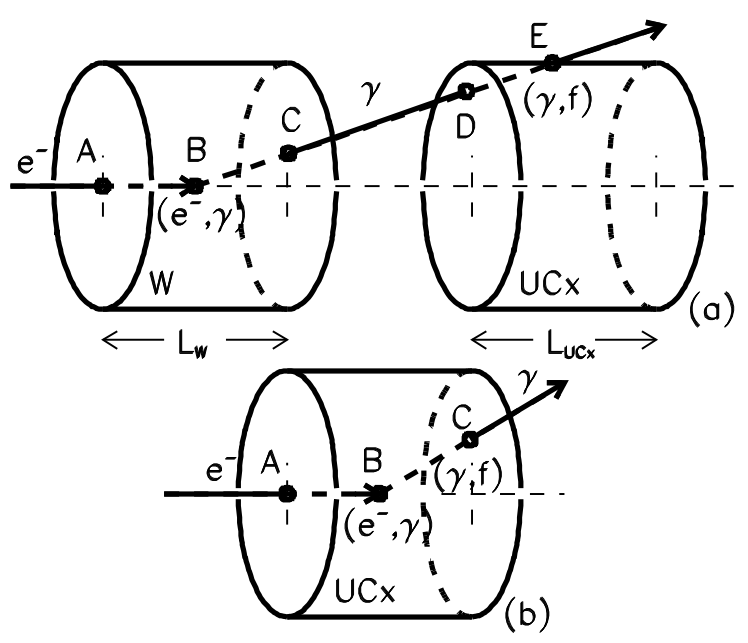

Figure 1: Two possible experimental configurations. (a) The intermediate converter produces the bremsstrahlung radiation. The initial electron beam reaches the $\mathrm{W}$ converter in the point $\mathrm{A}$, produces a gamma ray in the point $\mathrm{B}$ at the depth $z$. The gamma ray is emitted on a straight trajectory and can be absorbed traveling the distance $d_{W}$ up to the exit point C. The gamma ray reaches the UCx target in the point $\mathrm{D}$ and travel the distance $d_{U C x}$ up to the point $\mathrm{E}$, with a chance to produce a fission event or to be absorbed. (b) The bremsstrahlung radiation is produced in the target itself. 
In Fig. 2, the spectra $d^{2} N / \mathrm{d} \Omega \mathrm{d} E_{\gamma}$ of the bremsstrahlung emerging at various directions from a thick $\mathrm{W}$ target for an incident electron energy of $60 \mathrm{MeV}$ are represented. Here $N$ is the number of photons, $E_{\gamma}$ is the gamma energy in $\mathrm{MeV}$. At the small angles $0^{\circ}$ and $5^{\circ}$, Monte-Carlo simulations [14] for $\mathrm{W}$ target of same thickness can be compared. As expected, the high energy bremsstrahlung yields are larger in the vicinity of a small region around the $0^{\circ}$ emergence angle. As specified in [14], the high energy bremsstrahlung can be emitted only by electrons that have lost little energy and, consequently, have not yet deflected much by multiple scattering. Generally, our forward direction values of the spectra agree with those of [14]. However, larger deviations appear in the high energy region, where $E_{\gamma}>0.9 E_{i n c}, E_{\text {inc }}$ being the energy of the beam. These differences can be understood as follows: in the high energy region, we used different methods to correct the cross section. In this paper, the analytical Fano formula is kept while in the cited work [14], an interpolation with experimental data was done. In Fig. 3, the same quantities are plotted for an incident electron energy of $30 \mathrm{MeV}$. Similar trends as in Fig. 2 are displayed, but the yield at $15 \mathrm{MeV}$ is lowered by a factor 3 . From these examples, we illustrated the degree of accuracy of our simulations. Some differences with the reference data can be due to the fact that, by the Monte-Carlo technique, the yields were computed for gamma distributions in the ranges $\left[0^{\circ}-0.5^{\circ}\right]$ and $\left[0^{\circ}-5^{\circ}\right]$, while our curves are at $0^{\circ}$ and $5^{\circ}$, precisely. Our interest is now focussed on initial energies of $50 \mathrm{MeV}$, because from considerations which are not of physical nature, it is expected that our future facility will deliver about $50 \mathrm{MeV}$ electron beam. The results concerning the $50 \mathrm{MeV}$ electron beam onto $\mathrm{W}$ are displayed in Fig. 4. It seems that this energy is in fact suitable for our purpose because the yields of the forward spectra are only less than a factor two lower in the vicinity of $E_{\gamma} \approx 15 \mathrm{MeV}$ than those obtained at $60 \mathrm{MeV}$ incident energy (and only at $0^{\circ}$, otherwise the differences are much smaller). This decrease is not dramatic, and we avoid the production of intense gamma yields in the $50-60 \mathrm{MeV}$ region. The spectra are presented for two different target thicknesses: twice the electron range and half the electron range. For the smaller thicknesses, the yields in forward direction are much greater than for larger thicknesses, while a opposite trend is present for larger emission angles. This represents an indication for the choice of the converter thickness because a such behavior suggests that the converter with a thickness smaller than the electron range can produce a greater number of fission events in the fissionable target. The next step is the study of the radiative bremsstrahlung emission from the UCx target, 
when the $50 \mathrm{MeV}$ electron beam impinges directly on the target (without converter). The ratio between the number of $\mathrm{C}$ atoms versus that of $\mathrm{U}$ is 2.5 and the density of the mixture is $3.6 \mathrm{~g} / \mathrm{cm}^{3}$. Using the formulae presented in the previous section, the range of $50 \mathrm{MeV}$ electrons in the mixture was calculated as about $14.5 \mathrm{~g} / \mathrm{cm}^{2}$, being not too different than that in the $\mathrm{W}$ converter. Accordingly, for a comparison purpose with the results corresponding to the $\mathrm{W}$ converter, the thickness was taken also 30 $\mathrm{g} / \mathrm{cm}^{2}$ (approximately twice the range). The spectra obtained for the UCx target are presented in Fig. 5. Practically, the forward direction spectra have the same shapes as those in the case of the $\mathrm{W}$ converter. Having in mind that focusing the electron beam directly on the fissionable target, the bremsstrahlung radiation emitted in all the directions enhances the fission events number, it can be expected that the neutron rich nuclei productivity of the source increases. By placing the intermediate converter, a large amount of gamma rays emitted at larger angles cannot reach the target. It can be also noted that the UCx spectra display almost the same yields as the W converters.

The number of fission events produced in the uranium target per incident electron can be determined in the two experimental configurations: with the intermediate converter and bombarding the uranium target directly with electrons. In the first case, the number of fission events is obtained by integrating all the $\gamma$-rays which emerge from the converter, hit the target and produce reactions:

$$
\begin{gathered}
Y=\frac{\rho_{\mathrm{W}}}{A_{\mathrm{W}} u} \int_{0}^{E_{i}} \mathrm{~d} E \int_{\Omega} \mathrm{d} \Omega \int_{0}^{L} \mathrm{~d} z \exp \left[-\mu_{\mathrm{W}}\left(E_{\gamma}\right) d_{\mathrm{W}}(\theta)\right] \frac{\mathrm{d}^{2} \sigma_{t}\left(\theta, E_{i}, z\right)}{\mathrm{d} E_{\gamma} \mathrm{d} \Omega} \times \\
\sigma_{b f}\left(E_{\gamma}\right) \frac{1}{A_{\mathrm{UCx}} u} \frac{1-\exp \left[-\mu_{\mathrm{UCx}}\left(E_{\gamma}\right) d_{\mathrm{UCx}}(\theta)\right]}{\mu_{\mathrm{UCx}}}
\end{gathered}
$$

where $d_{\mathrm{W}}$ and $d_{\mathrm{UCx}}$ are the distances traveled by the gamma-ray (considered to have a straight line trajectory) in the converter and in the target, respectively, leading to an attenuation of the beam with the coefficients $\mu_{\mathrm{W}}$ and $\mu_{\mathrm{UCx}}, \sigma_{b f}$ is the cross section for photo-fission of ${ }^{238} \mathrm{U}$. The number of fission events obtained when the electron beam hits directly on the UCx target is determined in a similar way, the photo-fission being produced by the gamma rays created inside the target:

$Y=\frac{\rho_{\mathrm{UCx}}}{A_{\mathrm{UCx}} u} \int_{0}^{E_{i}} \mathrm{~d} E \int_{\Omega} \mathrm{d} \Omega \int_{0}^{L} \mathrm{~d} z \frac{\mathrm{d}^{2} \sigma_{t}\left(\theta, E_{i}, z\right)}{\mathrm{d} E_{\gamma} \mathrm{d} \Omega} \sigma_{b f}\left(E_{\gamma}\right) \frac{1}{A_{\mathrm{UCx}} u} \frac{1-\exp \left[-\mu_{\mathrm{UCx}}\left(E_{\gamma}\right) d_{\mathrm{UCx}}(\theta)\right]}{\mu_{\mathrm{UCx}}}$

These formulae give the number of fission events per incident electron for different geometries converter-targets and incident energies. In Fig. 6, the number of fission events produced with a $4 \mathrm{~mm}\left(7.5 \mathrm{~g} / \mathrm{cm}^{2}\right)$ thick W converter positioned at 4 and $8 \mathrm{~cm}$ distance from the target that with a $15 \mathrm{~mm}\left(30 \mathrm{~g} / \mathrm{cm}^{2}\right)$ thick $\mathrm{W}$ converter and that 


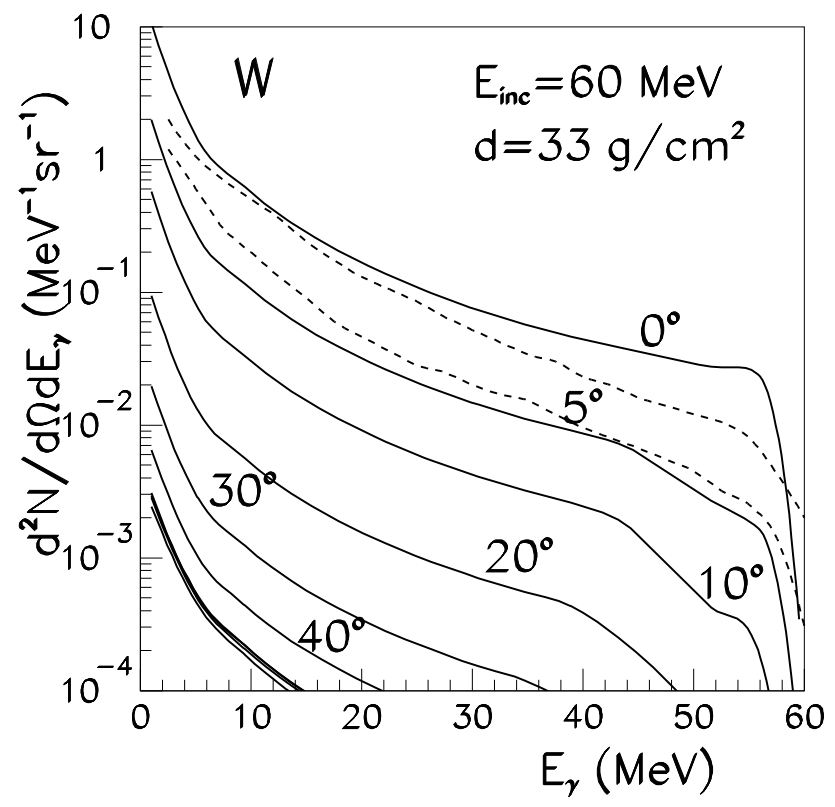

Figure 2: Angular and energy distributions of the bremsstrahlung emission per $60 \mathrm{MeV}$ incident electron. The cylindrical converter is made from $\mathrm{W}$, the thickness is $33 \mathrm{~g} / \mathrm{cm}^{2}$. The diameter is equal to the thickness. The full lines correspond to the present calculations. Some emission angles are marked on the plot. The results corresponding to $50^{\circ}$ up $80^{\circ}$, in steps of $10^{\circ}$, are also displayed but the yields are lower. The dotted lines are extracted from the work of Ref. [14] for $(0-0.5)^{\circ}$ and $(0-5)^{\circ}$. 


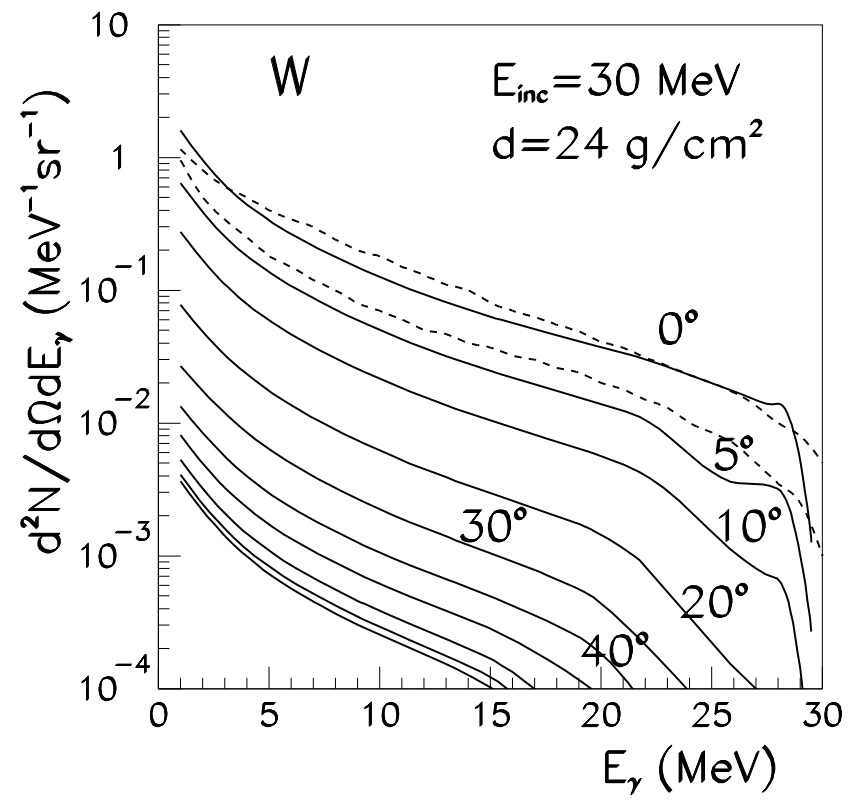

Figure 3: Angular and energy distributions of the bremsstrahlung emission per $30 \mathrm{MeV}$ incident electron. The cylindrical converter is made from W, the thickness is $24 \mathrm{~g} / \mathrm{cm}^{2}$. The diameter is equal to the thickness. The full lines correspond to the present calculations. Some emission angles are marked on the plot. The results corresponding to $50^{\circ}$ up $80^{\circ}$, in steps of $10^{\circ}$ are also displayed but the yields are lower. The dotted lines are extracted from the work of Ref. [14] for $(0-0.5)^{\circ}$ and $(0-5)^{\circ}$. 


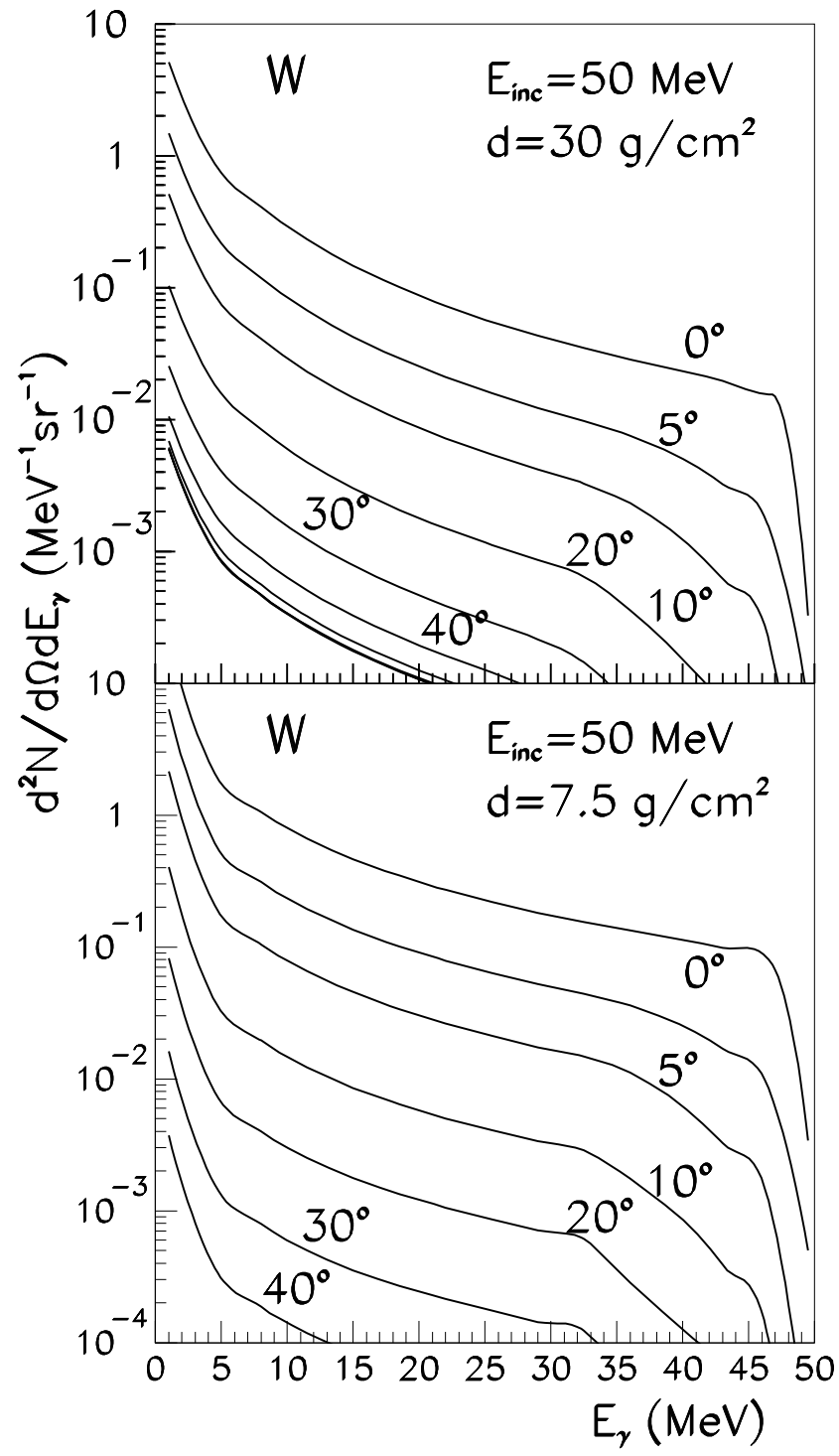

Figure 4: Angular and energy distributions of the bremsstrahlung emission per $50 \mathrm{MeV}$ incident electron. The cylindrical converter is made from W. Bottom: the thickness is $30 \mathrm{~g} / \mathrm{cm}^{2}$. Down: the thickness is $7.5 \mathrm{~g} / \mathrm{cm}^{2}$. The diameter is equal to approximately $1.5 \mathrm{~cm}\left(30 \mathrm{~g} / \mathrm{cm}^{2}\right)$. The full lines correspond to the present calculations. Some emission angles are marked on the plot. The results corresponding to $50^{\circ}$ up $80^{\circ}$, in steps of $10^{\circ}$ are also displayed but the yields are lower. 


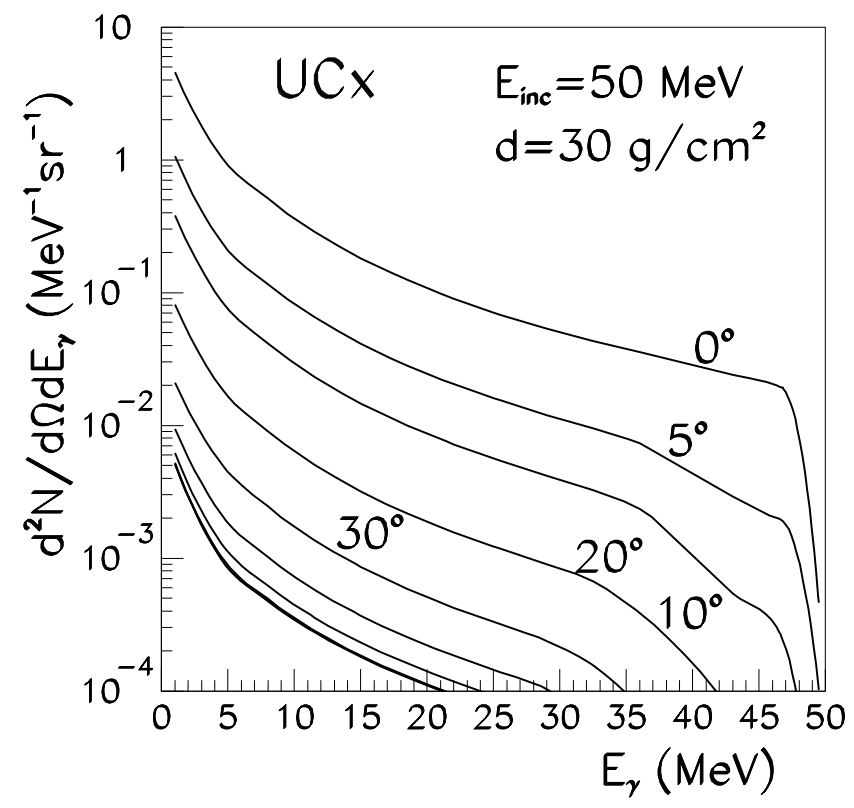

Figure 5: Angular and energy distributions of the bremsstrahlung emission per $50 \mathrm{MeV}$ incident electron. The cylindrical converter is made from $\mathrm{UCx}$, the thickness is $30 \mathrm{~g} / \mathrm{cm}^{2}$. The diameter is equal to the thickness. The full lines correspond to the present calculations. Some emission angles are marked on the plot. The results corresponding to $40^{\circ}$ up $80^{\circ}$, in steps of $10^{\circ}$ are also displayed but the yields are lower. 


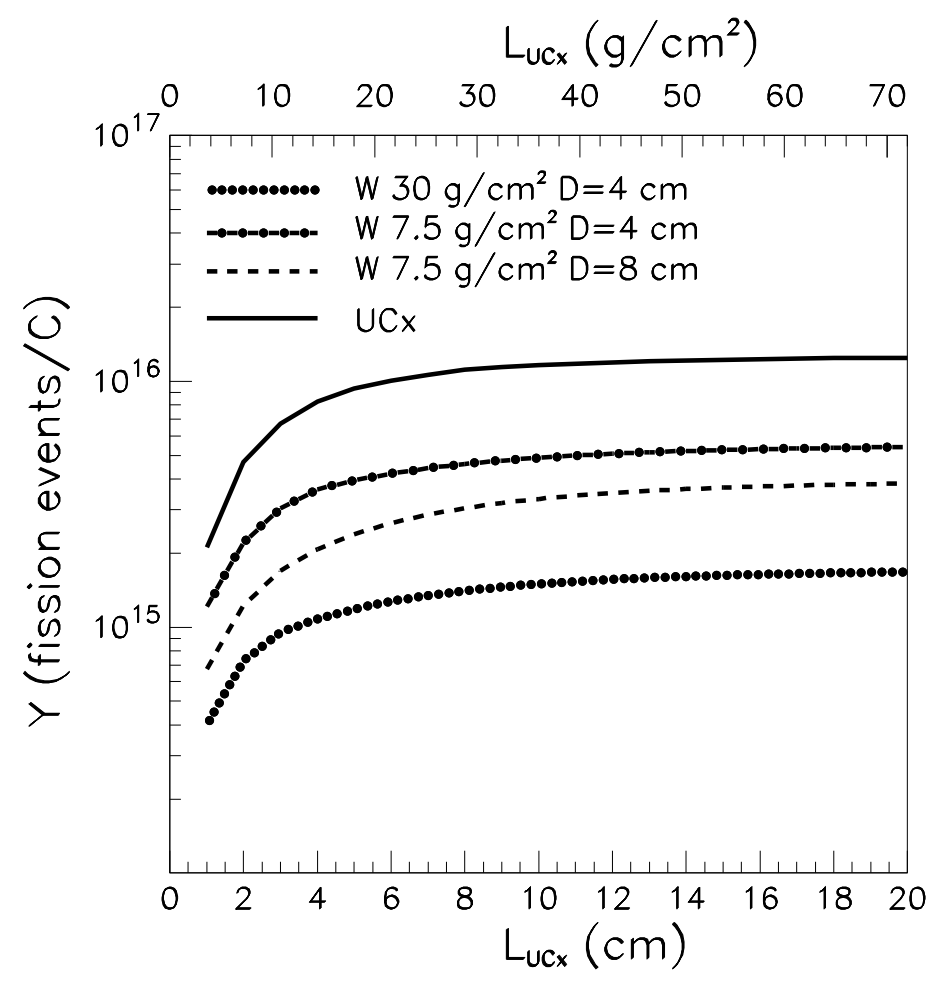

Figure 6: Number of fission events $Y$ per incident $50 \mathrm{MeV}$ electron Coulomb unity in the target as function of its thickness. The radius of the UCx target is $0.7 \mathrm{~cm}$. Four configurations are analyzed: a $30 \mathrm{~g} / \mathrm{cm}^{2} \mathrm{~W}$ converter (approximately twice the electron range) at $4 \mathrm{~cm}$ from the target, a 7.5 $\mathrm{g} / \mathrm{cm}^{2} \mathrm{~W}$ converter (approximately half the range) placed at 4 and $8 \mathrm{~cm}$ and the set-up without converter.

produced without converter for different lengths of the UCx target are presented. In all the configurations, the number of fission events increase with the UCx thickness, up a value of $8 \mathrm{~cm}$. After this point, the slope becomes flat and a plateau can be seen. Also, the number of fission events increases if the converter is positioned closer to the target.

The results show clearly that the yields increase when the experimental configuration without the intermediate converter is used. An experiment [25] for the production of radioactive beams was realized by some of the present authors with an $50 \mathrm{MeV}$ electron initial electron beam delivered by the LEP Pre-injector at CERN, using approximately the same conditions at those used in the present simulations. Gaseous elements produced as fission products emitted by the uranium carbide are trapped 


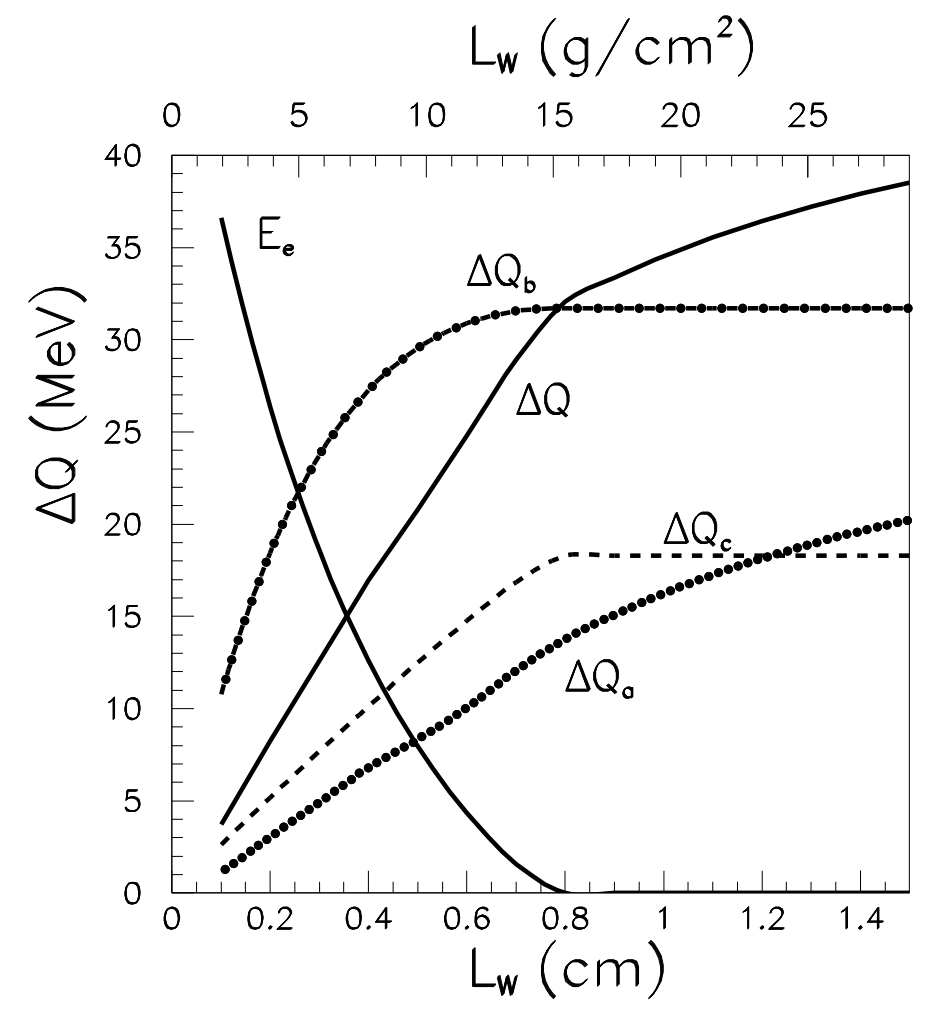

Figure 7: The quantities $\Delta_{c}, \Delta_{b}, \Delta_{a}$, and $\Delta Q$ per incident $50 \mathrm{MeV}$ electron as function of the W target thickness. The mean energy of the electron as function of the depth is referred as $E_{e}$

on a cryogenic cold finger. Their resulting activities were measured by gamma spectroscopy. Comparison with simulations can be realized in the assumption that the number of isotopes detected is directly proportional with the number of fission events. The following converter - target combinations were experimentally studied: a $4 \mathrm{~mm}$ thickness $\mathrm{W}$ converter positioned at 8 and $4 \mathrm{~cm}$ in front of the target and without converter. The ratio between the number of ${ }^{137} \mathrm{Xe}$ detected in the configuration of the converter placed at $4 \mathrm{~cm}$ with that of the converter at $8 \mathrm{~cm}$ was obtained experimentally 1.63 while our calculations give 1.41 . The ratio between the number of fission events of the configuration without converter with that of the converter placed at $4 \mathrm{~cm}$ is 2.96 experimentally and 2.3 in the simulations.

The energy released in the targets by the initial electron beam is given mainly by two processes: the collision energy loss from electron slowing down and the gamma attenuation in the material of the nascent bremsstrahlung radiation. This parameter 


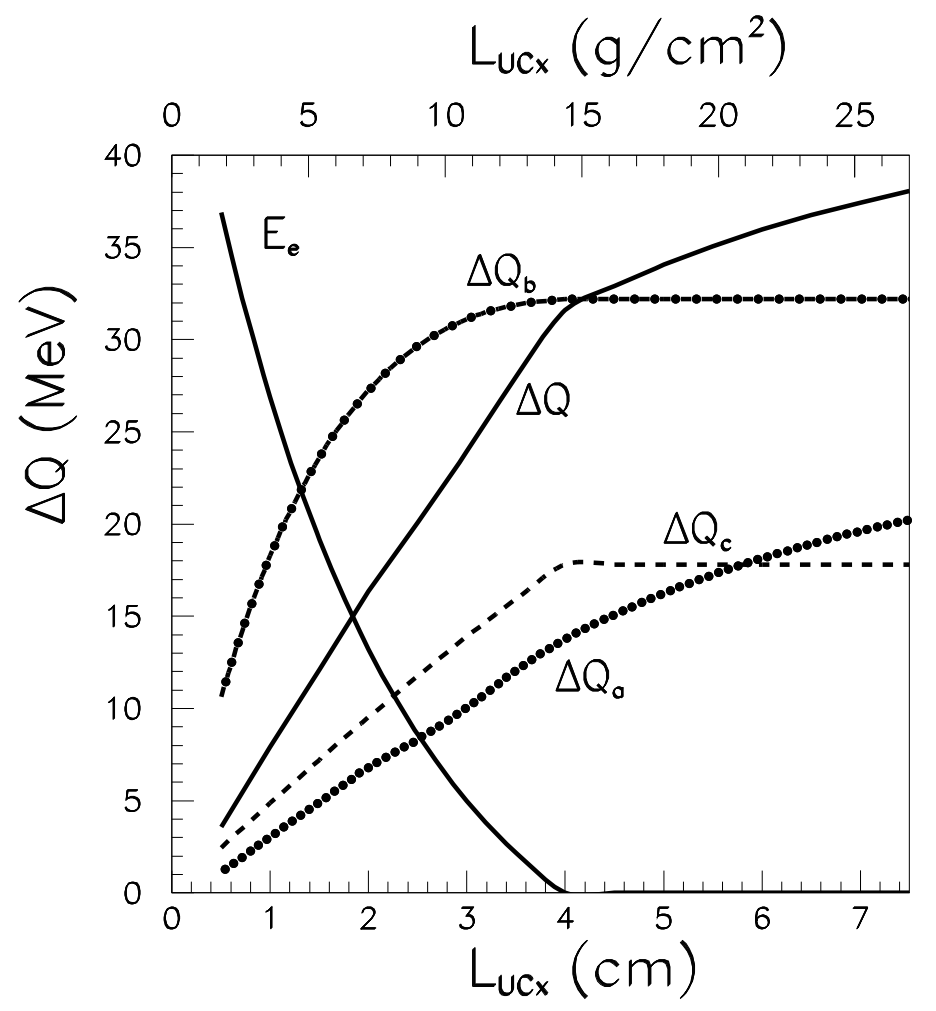

Figure 8: The quantities $\Delta_{c}, \Delta_{b}, \Delta_{a}$, and $\Delta Q$ per incident $50 \mathrm{MeV}$ electron as function of the UCx target thickness. The mean energy of the electron as function of the depth is referred as $E_{e}$ 


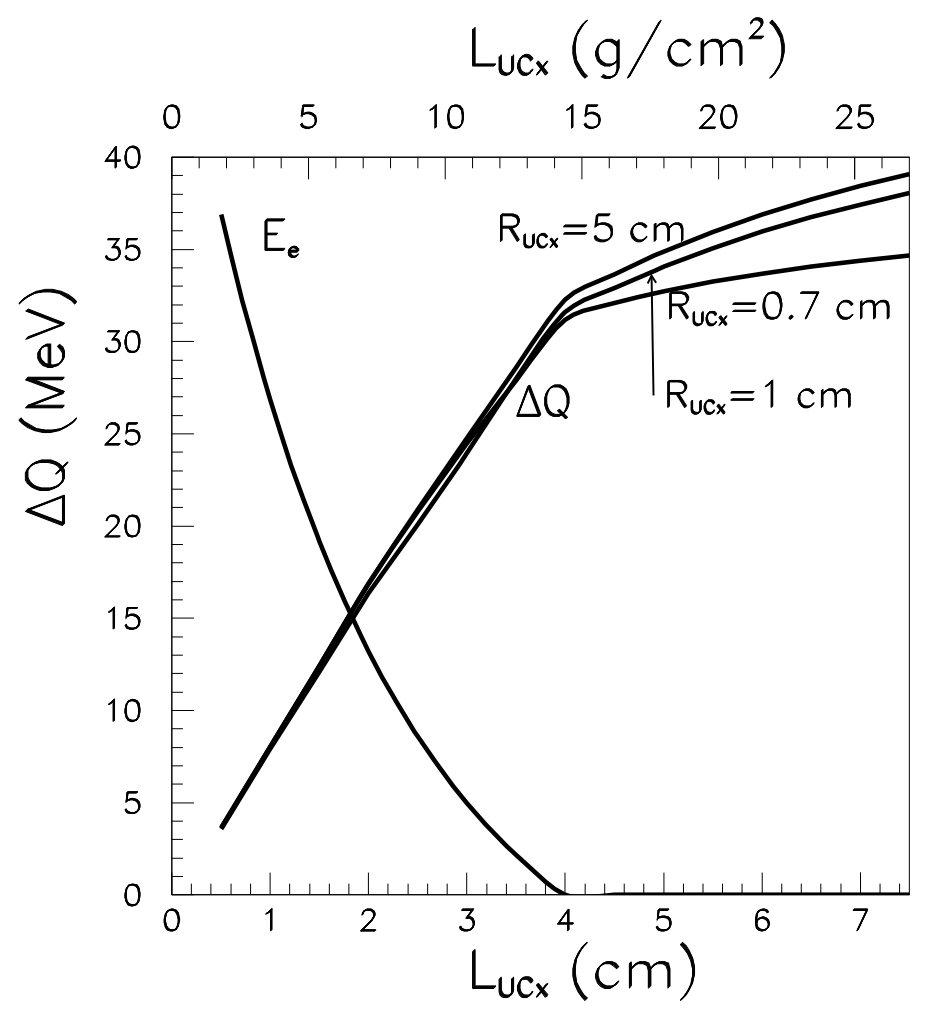

Figure 9: The quantity $\Delta Q$ per incident $50 \mathrm{MeV}$ electron as function of the UCx target thickness for different radius. The mean energy of the electron as function of the depth is referred as $E_{e}$ 


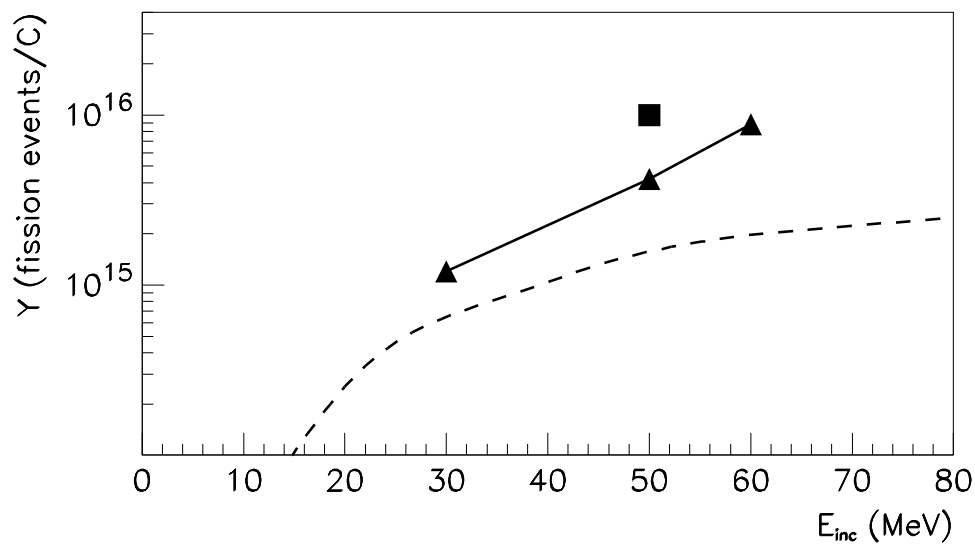

Figure 10: The calculated fission events numbers produced by charge unity of the incident particle as function of the incident energy of the beam. The dashed line represent the numbre of fission events with deuteron incident particles while the filled triangles correspond to electron with intermediate converter and the filled square to the configuration without intermediate converter.

has a crucial importance in the design of the source in order to avoid failures due to the heating. To compute the energy collision loss, the first step is to evaluate the energy of the electron $\bar{E}(z)$ as function of the depth $z$. For this purpose, the next equation is solved

$$
z=\int_{\bar{E}(z)}^{E_{i}}\left(\frac{\mathrm{d} \bar{E}}{\mathrm{~d} z}\right)^{-1} \mathrm{~d} E
$$

Now, the energy loss $\Delta Q_{c}$ due only to collisions or the energy loss $\Delta Q_{b}$ due only to bremsstrahlung emission as function of the distance traveled by the electron in the targets can be expressed as:

$$
\begin{aligned}
& \Delta Q_{c}=\int_{0}^{z} \frac{\mathrm{d} \bar{E}_{c}(z)}{\mathrm{d} z} \mathrm{~d} z \\
& \Delta Q_{b}=\int_{0}^{z} \frac{\mathrm{d} \bar{E}_{b}(z)}{\mathrm{d} z} \mathrm{~d} z
\end{aligned}
$$

The quantity $\Delta Q_{c}$ will be released to a target as heating. A part of the quantity $\Delta Q_{b}$ will be also released to the target as gamma-ray attenuation. The fraction $\Delta Q_{a}$ of the emitted bremsstrahlung energy released to the target by attenuation can be estimated by subtracting the total bremsstrahlung emission of a target with a given dimension in the presence of the attenuation from the total bremsstrahlung emission in the same 
geometrical conditions but computed neglecting the attenuation:

$$
\begin{gathered}
\Delta Q_{a}=\int_{0}^{E_{i}} E \mathrm{~d} E \int_{\Omega} \mathrm{d} \Omega \frac{1}{A u} \int_{0}^{L} \mathrm{~d} z \frac{\mathrm{d}^{2} \sigma_{t}\left(\theta, E_{i}, z\right)}{\mathrm{d} E_{\gamma} \mathrm{d} \Omega} \\
-\int_{0}^{E_{i}} E \mathrm{~d} E \int_{\Omega} \mathrm{d} \Omega \frac{\mathrm{d}^{2} N_{\gamma}}{\mathrm{d} E_{\gamma} \mathrm{d} \Omega}
\end{gathered}
$$

where the distribution of $N_{\gamma}$ is given by the Rel. (70). The total energy released to the follows as:

$$
\Delta Q=\Delta Q_{c}+\Delta Q_{a}
$$

In Figs. 7 and 8 the quantities $\Delta Q_{c}, \Delta Q_{a}, \Delta Q_{b}$ and $\Delta Q$ in $\mathrm{MeV}$ per incident $50 \mathrm{MeV}$ electron are represented as function of the target thickness for $\mathrm{W}$ and UCx targets, respectively. The both target radii are $1 \mathrm{~cm}$. The energy released to the target increases very rapidly up the range of the electron is reached. Increasing furthermore the target thickness, the released energy is due only to the gamma attenuation phenomena, and the slope of $\Delta Q$ decreases. Approximately $35 \mathrm{MeV}$ of the incident electron energy are lost in the target. In Fig. $9, \Delta Q$ is plotted for several values of the $\mathrm{UCx}$ target radius. The slopes for the increase of $\Delta Q$ are practically the same in the three cases up the thickness reaches the electron range, and even afterwards, the change of the radius does not produce too drastic variations. This effect is a direct consequence of the bremsstrahlung angular distribution behavior, the reactions produced by the bremsstrahlung radiations being mainly confined in the vicinity of the target symmetry axis.

It is usefull to compare the number of fission events obtained for a given geometry but for different incident energies of the incoming beam. In Fig. 10, the number of fission events produced in a $6 \mathrm{~cm} \mathrm{UCx}$ target (radius $=0.7 \mathrm{~cm}$ ) are displayed for different incident energies. The filled triangles correspond to the configuration with the intermediate converter placed at $4 \mathrm{~cm}$ in front of $\mathrm{UCx}$, and the thickness of the converter is half the range of the electron at the specified incident energy. The configuration without intermediate converter for $50 \mathrm{MeV}$ electrons is plotted with a filled square. The results show that the configuration without converter enables the best production of fission events. Using the photo-neutron cross sections of Ref. [26], in the case of the configuration without converter, the number of neutrons created inside the target was estimated to be $3.2 \times 10^{16}$ neutrons per incident charge unity. That means, the number of neutrons created inside the target by direct reactions is approximately 3.2 times larger than the number of fission events. On the same figure, with dashed line the productions expected for deuteron incident particle are plotted. The UCx target 
has the same dimensions and a Carbon converter is placed at $1 \mathrm{~cm}$ from the target. From the theoretical point of view, a similar kind [4] of modelisation is used, based on the knowledge of the angular and energy distributions of the fast neutrons produced in the converter (through the deuteron break-up reaction) and the neutron induced fission cross sections. This exploratory analyzis shows that the photo-fission allows the production of fission events yields which can compet with those obtained in the frame of fast neutron induced fission concept. Investigations stressing similar ideeas concerning the oportunity of the concept based on bremsstralung induced fission are reported in Ref. [27].

Finally, this study gives evidence for the advantages obtained by eliminating the intermediate converter and offers the possibility to estimate the required radiative shield of our combination [28]. Furthermore, the numerical code developed will permit the evaluation the efficiency dependence for different quantities of the neutron rich ion source (such as the number of fission events) in order to optimize the yields.

\section{Acknowledgement}

This work was sponsored by the European Contracts SPIRAL II No. ERB 4062 PL 975009, No. FMGE CT 980100 and by the IDRANAP European Center of Excellence.

\section{References}

[1] J.A. Nolen, in: D.J. Morissey (Ed), 3rd International Conference on Radioactive Nuclear Beams, Edition Frontieres, Gif-sur-Yvette, 1993, p. 111.

[2] F. Clapier, A.C. Mueller, C. Obert, O. Bajeat, M. Ducourtieux, A. Ferro, A. Horbowa, L. Kotfila, C. Lau, H. Lefort, S. Kandri-Rody, N. Pauwels, J.C. Poitier, J. Proust, J.C. Putaux, C.F. Liang, P. Paris, A.C.C. Vilari, R. Lichtenhaler, L. Maunoury, J. Lettry, Phys. Rev. ST Accel. Beams 1 (1988) 013501.

[3] S. Kandri-Rody, J. Obert, E. Cottereau, O. Bajeat, M. Ducourtieux, C. Lau, H. Lefort, J.C. Potier, J.C. Putaux, F. Clapier, J. Lettry, A.C. Mueller, N. Pauwels, J. Proust, C.F. Liang, P. Paris, H.L. Ravn, B. Roussiere, J. Sauvage, J.A. Scarpaci, F. Le Blanc, G. Galu, I. Lhenry, T. Von Egidy, R. Antoni, Nucl.Instr.Meth. B 160 (200) 1. 
[4] M. Mirea, O. Bajeat, F. Clapier, F. Ibrahim, A.C. Mueller, N. Pauwels, J. Proust, Eur. Phys. J. A 11 (2001) 59.

[5] W.T. Diamond, Nucl. Instr. Meth. A 432 (1999) 471.

[6] H. Ries, G. Mank, J. Drexler, R. Heil, K. Huber, U. Kneissl, R. Ratzek, H. Stroher, T. Weber, W. Wilke, Phys. Rev. C 29 (1984) 2346.

[7] J.T. Caldwell, E.J. Dowdy, B.L. Berman, R.A. Alvarez and P. Meyer, Phys. Rev. C 21 (1980) 1215.

[8] C. Cetina, P. Heimberg, B.L. Berman, W.J. Briscoe, G. Feldman, L.Y. Murphy, H. Crannel, A. Longhi, D.I. Sober, J.C. Sanabria and G.Ya. Kezerashvili, Phys. Rev. C 65 (2002) 044622.

[9] Y.S. Tsai, V. Whitis, Phys. Rev. 149 (1966) 1248.

[10] G. Knop, W. Paul, in: K. Sieghbahn (Ed.), Alpha-, Beta- and Gamma-Ray Spectroscopy, North Holland Publ. Co. Amsterdam, 1968 chap. 1, p. 1.

[11] W.A. McKinley Jr., H. Feshbach, Phys. Rev. 74 (1948) 1759.

[12] W.T. Scott, Rev. Mod. Phys. 35 (1963) 231-313.

[13] H.W. Koch, J.W. Motz, J.W., Rev. Mod. Phys. 31 (1950) 920.

[14] M.J. Berger, S.M. Seltzer, Phys. Rev. C 2 (1970) 621.

[15] S.P. Ahlen, Rev. Mod. Phys. 52 (1980) 121-173.

[16] R.M. Sternheimer, Phys. Rev. 88 (1952) 851.

[17] L. Pages, E. Bertel, H. Joffre, L. Sklavenitis, At. Data 4 (1972) 1.

[18] T. Tabata, R. Ito, Nucl. Instr. Meth. 158 (1979) 521.

[19] Y. Mejaddem, D. Belkic, S. Hyodynmaa, A. Brahme, Nucl. Instr. Meth. B 173 (2001) 397.

[20] Y.-S. Tsai, Rev. Mod. Phys. 46 (1974) 815.

[21] W.R. Leo, In: Techniques for Nuclear and Particle Physics Experiments, Springer Verlag Berlin, Heildelberg, New York, 1987, chap. 2, p. 17.

[22] R.M. Sternheimer, in: L.C.L. Yuan, C.S. Wu, (Eds.), Methods of Experimental Physics, Academic Press, New York, 1961, Vol. 5, Part A, chap. 1.1, p. 1.

[23] C.M. Davisson, 1968. in: K. Sieghbahn (Ed.), Alpha-, Beta- and Gamma-Ray Spectroscopy, North Holland Publ. Co. Amsterdam, 1968, appendix 1, p. 827. 
[24] M. Mirea, O. Bajeat, F. Clapier, N. Pauwels, J. Proust, Preprint IPNO 99-06, 1996.

[25] F. Ibrahim, J. Obert, O. bajeat, J.M. Buhour, F. Clapier, C. Donzaud, M. Ducourtieux, J.M. Dufour, S. Essabaa, S. Gales, D. Guillemaud-Mueller, F. Hosni, O. Hubert, A. Joinet, U. Koster, C. Lau, H. Lefort, G. Le Scornet, J. Lettry, A.C. Mueller, M. Mirea, N. Pauwels, O. Perru, J.C. Poitier, J. Proust, F. Pougheon, H. Ravn, L. Rinolfi, G. Rossat, H. Safa, M.G. Saint Laurent, M. Santana-Leitner, O. Sorlin, D. Verney, Preprint EURISOL, http://www.ganil.fr/eurisol/.

[26] B.L. Berman, At. Data. Nucl. Data. Tables 15 (1975) 319.

[27] Yu.Ts. Oganessian, S.N. Dmitriev, J. Kliman, O.A. Maslov, G.Ya. Starodub, A.G. Belov, S.P. Tretiakova, Nucl. Phys. A 701 (2002) 87c.

[28] M. Mirea, O. Bajeat, F. Clapier, M. Hassaine, F. Ibrahim, A.C. Mueller, N. Pauwels, J. Proust, D. Verney, R. Antoni, L. Bourgois, S. Kandri-Rody, submitted Radioprotection. 